\title{
Monolithic hemispherical microlenses fabricated by selective oxidation of AlGaAs
}

\section{Yong Xu, Chuan-Cheng Cheng, Reginald K. Lee, John D. O'Brien, Amnon Yariv, et al.}

Yong Xu, Chuan-Cheng Cheng, Reginald K. Lee, John D. O'Brien, Amnon Yariv, Axel Scherer, "Monolithic hemispherical microlenses fabricated by selective oxidation of AIGaAs," Proc. SPIE 3279, Light-Emitting Diodes: Research, Manufacturing, and Applications II, (7 April 1998); doi: $10.1117 / 12.304423$

SPIE Event: Optoelectronics and High-Power Lasers and Applications, 1998, San Jose, CA, United States 


\title{
Monolithic hemispherical microlenses fabricated by selective oxidation of AlGaAs
}

\author{
Y. Xu, C.C. Cheng, R.K. Lee, J. O'Brien ${ }^{a}$, A. Yariv and A. Scherer \\ Applied Physics and Electrical Engineering \\ California Institute of Technology, MS 128-95 \\ Pasadena, CA 91125 USA \\ ${ }^{a}$ Electrical Engineering / Electrophysics \\ University of Southern California, mc-0271 \\ Los Angeles, CA 90089 USA
}

\begin{abstract}
We have developed a new method for the fabrication of monolithic AlGaAs microlenses on the surface of GaAs/AlGaAs light emitting diodes by combining crystal growth, ion etching and steam oxidation with wet chemical removal of the oxide. Control over the precise processing parameters has resulted in the precise control over the shape, radius, position and smoothness of the microfabricated hemispheres. These microlenses can readily be used for the fabrication of highly efficient light-emitting diodes.
\end{abstract}

Keywords: semiconductor laser, Schottky barrier diode, electrostatic current confinement, MESFET

\section{INTRODUCTION}

The efficiency of semiconductor light-emitting diodes has traditionally been limited by the output coupling efficiency of the light from the semiconductor to air. From a planar GaAs LED, total internal reflection typically limits the exit angle over which light can be coupled from the surface of the semiconductor wafer to approximately $17^{0}$ from the surface normal. This results in a relatively poor external diode efficiency of only a few percent. The most successful, although also relatively expensive method of improving the output coupling efficiency has been to use a vertical cavity laser structure which enhances light emission in modes perpendicular to the wafer surface, and thus results in quantum efficiencies above $50 \% .^{1,2}$ Removal of the substrate and roughening of the semiconductor surface by texturing have also been successfully applied towards improving the external quantum efficiencies of light-emitting diodes to about $30 \% .^{3}$

For commercial diodes, transparent hemispherical or dome lenses of plastic or high-index glass materials are commonly mounted onto the light-emitting surface, thereby improving the output coupling efficiency and also for controlling the directionality of the light output. ${ }^{4}$ Since, for maximum effectiveness, the active light-emitting material must be located at the center of the hemisphere and be intimate contact with the device surface, this technology is generally limited to relatively large dome sizes. An alternative approach which was recently explored uses lithographically fabricated photoresist domes, ${ }^{5}$ whose hemispherical shape is transferred from the polymer into the underlying substrate by ion milling. This technique relies on a high quality of the etched dome surface, and low selectivity of the etch rate of the semiconductor versus the polymer mask. Alternatively, transparent high index domes have been formed by a purely lithographic technique. UV exposure of thick $A s_{2} S_{3}$ layers uses the self-developing properties of this material to fabricate hemispheres, thereby avoiding the semiconductor etching process. Here, we describe an alternative approach to lithographically fabricated hemispherical lenses. Using this fabrication technique, lenses can be monolithically integrated and self-aligned onto the light-emitting diodes. Furthermore, the crystal grown structure allows accurate control over the size, and shape of the microlenses. We have also developed techniques for self-aligning these microlenses with underlying light-emitting active regions for the definition of ultra-small light-emitters.

Send correspondence to $\mathrm{Y} . \mathrm{Xu}$

Y.X.: Email: yong@cco.caltech.edu; Telephone: 626-395-4413; Fax: 626-405-0928

SPIE Vol. $3279 \bullet 0277-786 \times / 98 / \$ 10.00$ 


\section{PROCEDURE}

The hemisphere fabrication technique relies on the conversion of a 2-dimensional "disk" pattern on the surface of the semiconductor into a 3-dimensional hemispherical lens. To provide the controlled pattern transfer in the third dimension, we introduce a rertical composition gradient during the epitaxial crystal growth of the active diode material. When proper fabrication parameters are chosen, the wet chemical etch rate and the steam oxidation rate are strongly dependent on the relative $\mathrm{Al}$ and $\mathrm{Ga}$ contents in the $\mathrm{AlGaAs}$, and reproducible 3-D hemispheres with smooth outer surfaces can be defined. In this study, we have investigated and compared fabrication approaches suitable for the definition of high quality self-aligned dome lens structures.

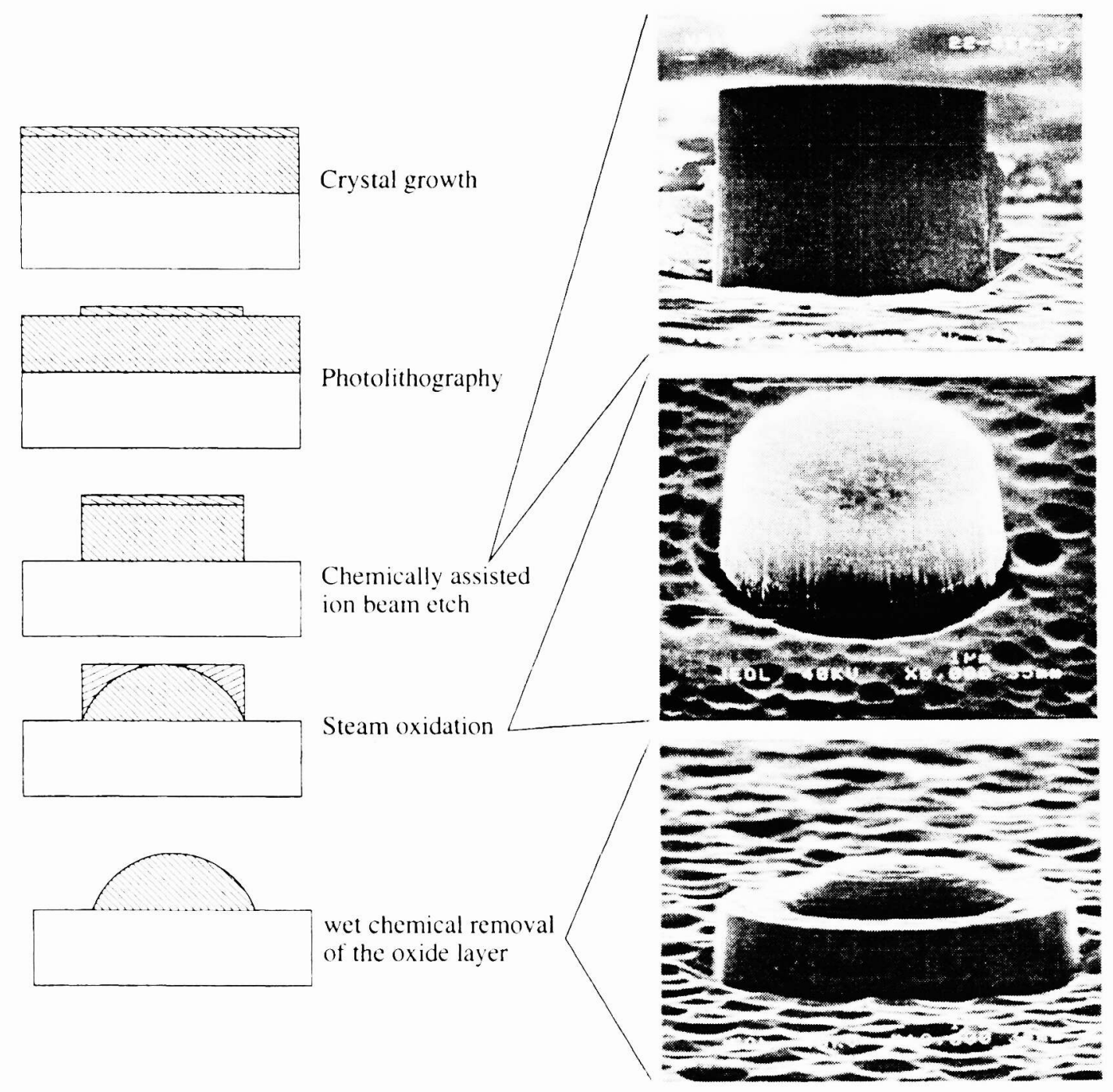

Figure 1. Process flow showing the fabrication of microlenses.

In all of our starting material, we introduce a Ga gradient in the AlAs laver above the light-emitting structure during molecular beam epitaxy (MBE) crystal growth. We vary the concentration from pure AlAs at the semiconductor surface to $10 \% \mathrm{Ga}$ at the lens/light emitter interface. A LED pillar is then defined by chlorine assisted ion etching by using a photolithographically defined circular etch mask through this graded AlGaAs layer. Following the anisotropic ion etch. the entire structure is oxidized by using steam oxidation in a tube furnace at temperatures between $300-400^{\circ} \mathrm{C}$. resulting in a aluminum oxide shell of varying thickness depending on the original Ga concentration. During this oxidation procedure. nitrogen gas is bubbled through a heated water bath before flowing 


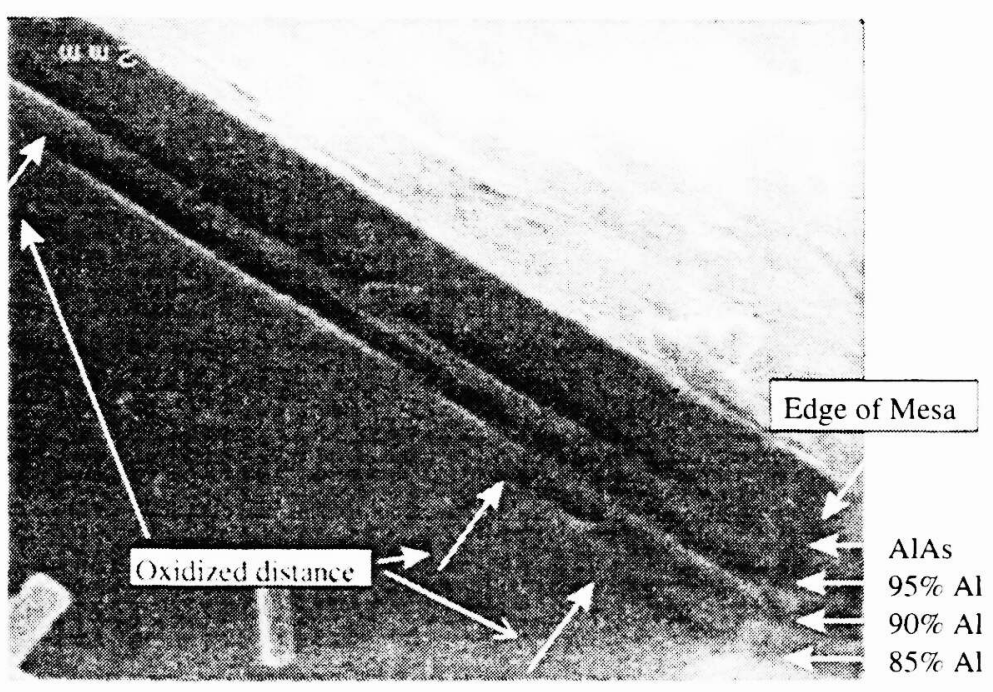

Figure 2. Electron micrograph showing the oxidation rate dependence on the aluminum concentration

through the resistively heated furnace tube containing the sample. In Figure 1, we schematically summarize the processing sequence for definition of hemispherical lenses. Thin compositional stratification layers of $5 \mathrm{~nm}$ of high Ga content AIGaAs were incorporated periodically during the growt of the graded AlGaAs layer to encourage the almost two-dimensional diffusion from the pillar circumference inwards. As a result, a high quality hemisphere of AlAs can be formed underneath a $\mathrm{Al}_{2} \mathrm{O}_{3}$ shell. The $\mathrm{Al}_{2} \mathrm{O}_{3}$ can then be selectively removed in the final processing step by rapid reheating of the oxide, followed by a wet chemical HF etch. This step is necessary if metal contacts must be used for electrical pumping of the active material. We find that immersion into dilute hydrofluoric acid under ultrasonic agitation can be used to remove the oxide almost entirely from the surface of the hemisphere. For optically pumped structures, such oxide removal is not necessary and may not be desirable, since the aluminum oxide provides with a good antireflection and passivation coating for the resulting optical devices.

This processing sequence can be significantly simplified by following the anisotropic CAIBE etch immediatelly with a chemical wet etch. The graded AlGaAs layer is then etched at predetermined rates, depending on the $\mathrm{Al}$ concentration, and the oxidation step can be avoided. In this simpler procedure, we immerse the sample with a surface pattern of etched pillars of graded AlGaAs into dilute HF to remove the AlGaAs. Again, stratification of the composition of the graded layer with thin GaAs layers is important to encourage two-dimensional etching of the AlGaAs in the chemical etch, to ensure that the rates are proportional to the aluminum concentration in the layers. This simple room-temperature procedure has also allowed us also to generate hemispheres, although the surface morphology of the resulting hemispheres indicates some selective etching along crystallographic planes, and precise process control is critical for good reproducibility. Thus, although this direct etching procedure may well be less complicated, higher quality hemispheres were obtained when combining the steam oxidation process with a subsequent chemical removal process. Figure 2 shows a schematic flow-diagram for this simpler fabrication procedure.

\section{RESULTS AND DISCUSSION}

In the microlens fabrication method described here, the Ga gradient in the hemisphere-defining layer ultimately controls the shape of the micromachined lens. The duration of the oxidation and original mask dimensions define the lateral radius and anisotropy of the dome. Ideally; precise control over both of these process parameters can allow the optimized design of output-coupling lenses for a light-emitter structure with predetermined light focussing characteristics. Within this process parameter space, however, the smoothness of the final lens profile can be optimized by the precise oxidation conditions (i.e., temperature and duration) as well as the chemical etching conditions used to reveal the lens structure from underneath the aluminum oxide. We have explored the parameter space for the microfabrication of these microlenses. and discuss some of our findings below. 


\subsection{Steam Oxidation}

To determine the aluminum concentration profile which results after oxidation in a high-quality hemispherical lens, a calibration of the oxidation rate as a function of the $\mathrm{Al}$ content has to be performed. Figure 2 shows a test sample which was used to calibrate this concentration-dependence of the oxidation rate. This sample initially consisted of a MBE-grown structure containing several AlGaAs epilayers with $85 \%, 90 \%$ and $95 \%$ aluminum. Mesas were formed on this sample after photolithography and CAIBE etching to expose the edges of these AlGaAs layers. Then, these layers were steam oxidized at $400^{\circ}$, and cleaved to reveal the extent of the AlGaAs oxidation from the outside surface. As can be seen from this figure, the oxidation distance strongly depends on the aluminum content of the initial epilayers. This oxidation behavior has in the past been well described and changes from reaction to diffusion limited growth with increased oxidation time and temperature. ${ }^{6}$ Our observations agree well with other previously published experimental results. ${ }^{7}$

\subsection{Oxide removal}

Once the steam oxidation process has been completed, the hemispherical lens is covered with a thick layer of aluminum oxide (Figure 3a). A chemical etch can be used to reveal semiconductor lens surface underneath the oxide. To accomplish this, the sample is reheated for a short time in the furnace and air cooled rapidly to "thermally shock" the oxide layer. Then, this layer can be removed by immersion in a $10 \% \mathrm{HF}$ bath. Figure $3 \mathrm{~b}$ shows a partially revealed lens, and it is observed that the oxide layer is cracking and delaminating from the semiconductor surface. We find that ultrasonic agitation is necessary to leave a clean surface, since otherwise pieces of the oxide remain on top of the lens structure (Figure 3c). The immersion time in the HF bath has to be carefully controlled, since this acid does attack the AlGaAs layer. Such chemical attack reduces the diameter of the microlens, as can be seen in Figure 4a, and leads to anisotropic facet formation in the lens as a result of the crystalline anisotropy in the chemical etch rate. Of course, the HF concentration can also be reduced, but this often leads to the evolution of terraces corresponding to the stratification layers introduced during the crystal growth, as can be observed in Figure $4 \mathrm{~b}$. Etching times of 10 seconds have provided us with our best results, which are shown in Figure $5 \mathrm{a}$ and b. In these scanning electron micrographs, it is possible to observe a relatively smooth lens surface.

\subsection{Lithography and Fabrication of Self-Aligned Diodes}

Lithography, ion etching, and planarization can also be used to self-align microlenses with electrically pumped active material. Figure 6 shows such a scheme, in which the same etched mesa can be used to define both microlens and oxide pumping aperture for a micro-LED. In such a structure, the active pumped material is self-aligned and positioned directly underneath the hemispherical lens, allowing the miniaturization of this light-emitting structure. The quality of the initial optical lithography used to create the etched mesas before steam oxidation of the microlenses always determines the lateral shape of these structures. If the perimeter of the lens contains notches or other defects, these are transferred during the oxidation process, and deteriorate the quality of the lens, as can be observed in Figure 6 . Thus, it is not only important to optimize the lithography and etching conditions, but it is also possible to design non-hemispherical lens shapes depending on the required application.

\section{CONCLUSION}

We have developed and optimized a new microlens fabrication technique for the fabrication of monolithic integration of hemishperical domes onto light-emitting diodes. Here, we have shown that it is possible to control the shape, size, position, and smoothness of the lenses by careful optimization of the fabrication parametersh, and show methods for applying these lenses on $\mathrm{GaAs}$ and $\mathrm{AlGaAs}$ light-emitting diodes. We believe that this microlens fabrication technique can also be applied to fabricating similar structures on the In GaP/InGaAlP visible light-emitting material, for which AlAs is also lattice-matched. We expect that this procedure will allow the fabrication of highly efficient LED's for communications and displays.

\section{ACKNOWLEDGEMENTS}

The authors wish to thank O. Painter for helpful discussions. This research was funded by the Army Research Office, the National Science Foundation and the Office of Naval Research which are also gratefully acknowledged. 


\section{REFERENCES}

1. G. Yang, M. MacDougal, and P. Dapkus, "Ultralow threshold current vertical-cavity surface-emitting lasers obtained with selective oxidation," Elect. Lett. 31, pp. 886-888, 1995.

2. K. Lear, K. Choquette, R. Schneider, S. Kilcoyne, and K. Geib, "Selectively oxidized vertical-cavity surfaceemitting lasers with 50-percent power conversion efficiency," Elect. Lett. 31, p. 208, 1995.

3. I. Schnitzer, E. Yablonovitch, C. Caneau, T. Gmitter, and A. Scherer, "30-percent external quantum efficiency from surface textured, thin-film light-emitting-diodes," App. Phys. Lett. 63, p. 2174, 1993.

4. S. Galginaitis J. App. Phys. 36, p. 460, 1965.

5. E. Strzelecka, G. Thompson, G. Robinson, M. Peters, B. Thiebeault, M. Mondry, V. Jayaraman, F. Peters, and L. Coldren, "Paper 2691-07," in SPIE Photonics West '96, 1996.

6. M. Ochiai, G. Giudice, H. Temkin, J. Scott, and T. Cockerill, "Kinetics of thermal-oxidation of AlAs in watervapor," App. Phys. Lett. 68, pp. 1898-1900, 1996.

7. K. Choquette, R. Schneider, K. Lear, and K. Geib, "Low threshold voltage vertical-cavity lasers fabricated by selective oxidation," Elect. Lett. 30, pp. 2043-2044, 1994. 

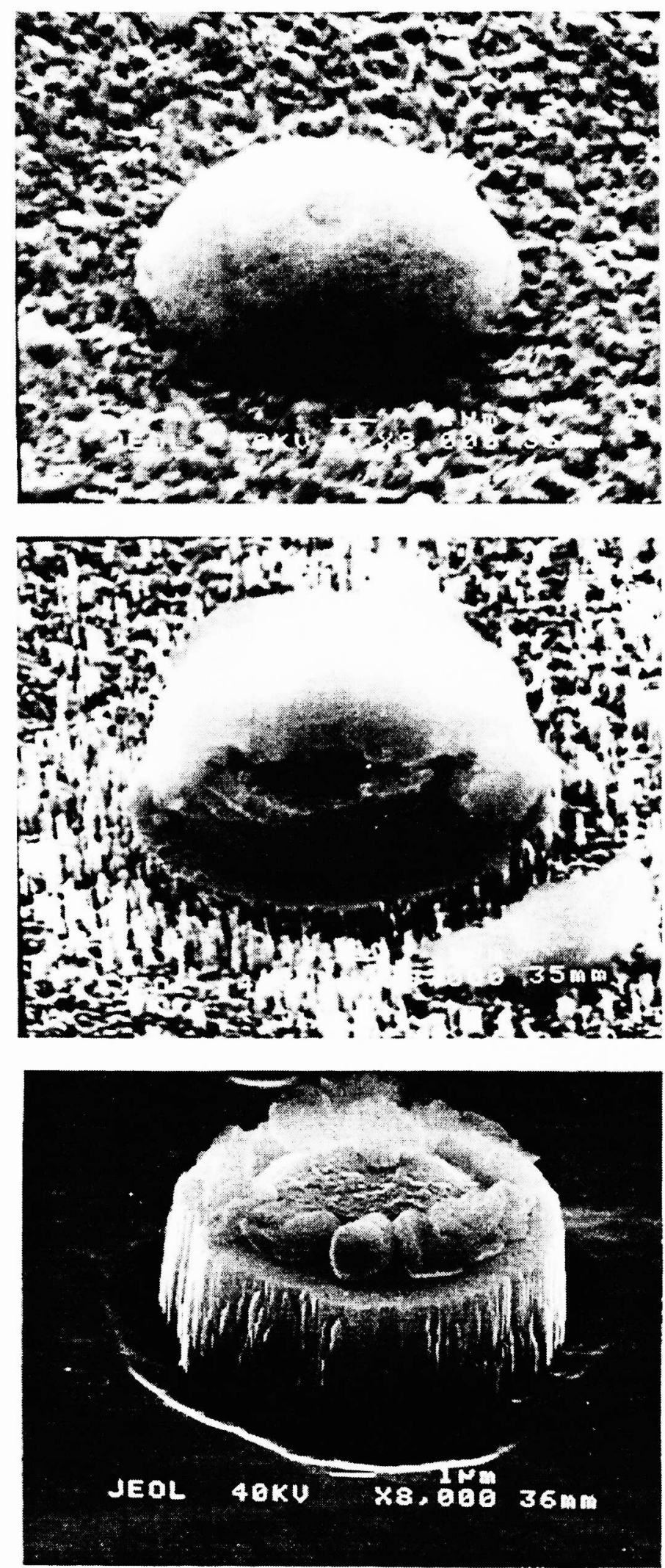

Figure 3. (a) top - Oxidized microlens before complete removal of the aluminum oxide. (b) middle - Partially revealed microlens. (c) bottom - Microlens after oxide removal without ultrasonic agitation. 

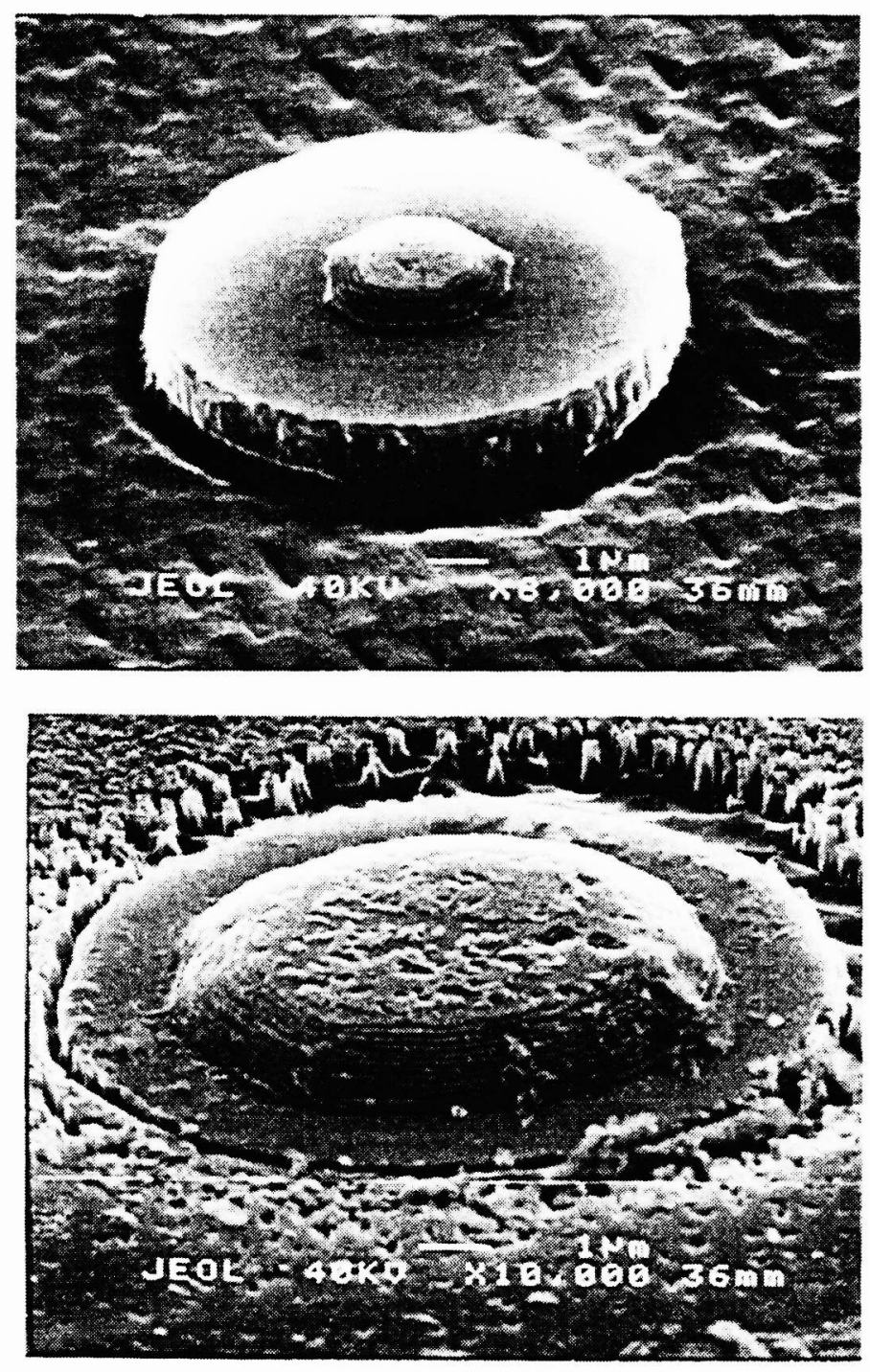

Figure 4. (a) top. Electron micrograph of overetched microlens. (b) bottom, Microlens showing terraces from the MBE grown stratification lavers. 

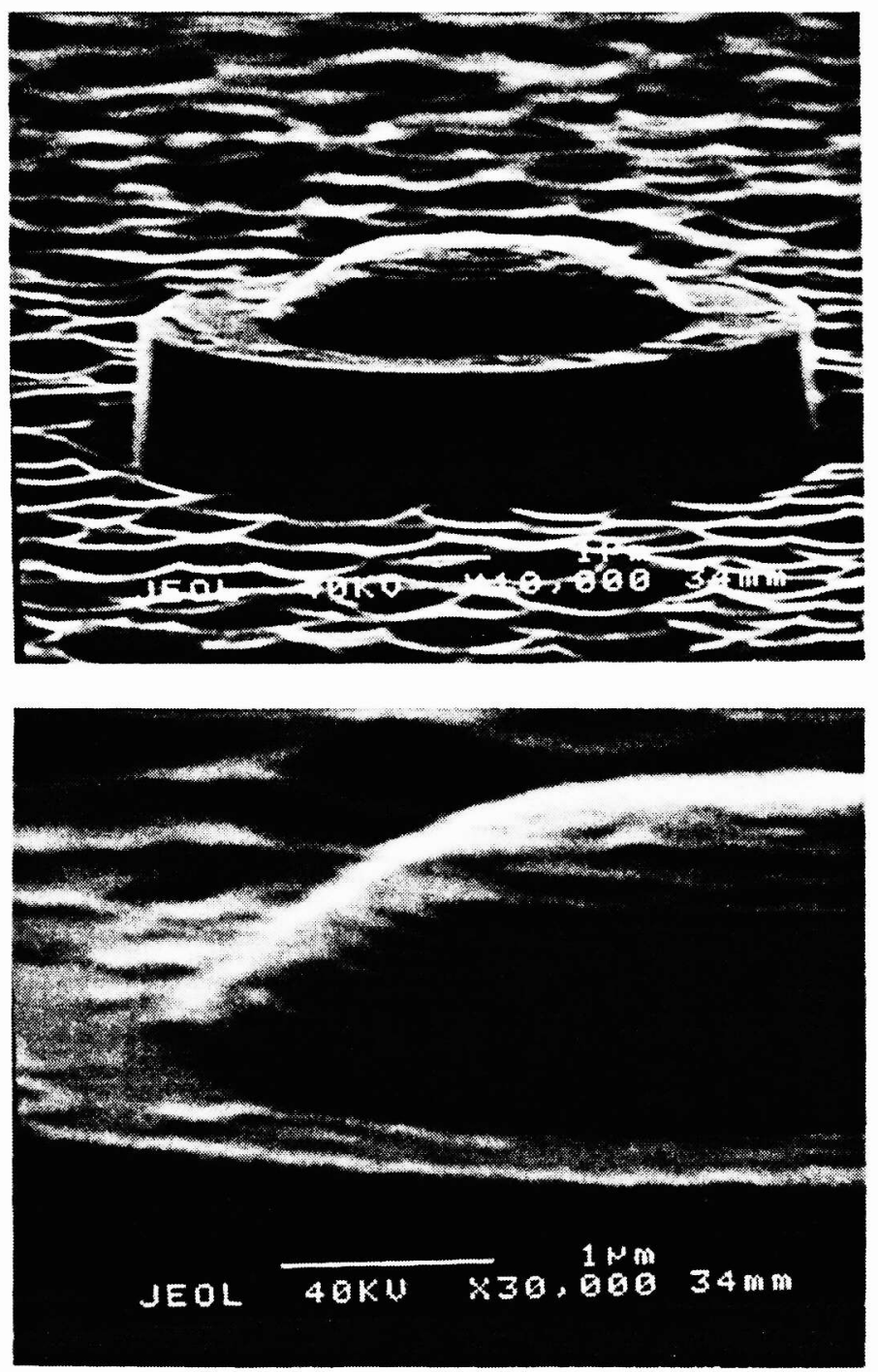

Figure 5. Top - Optimally fabricated hemispherical microlens (a). Bottom - an enlargement of the lens perimeter showing surface roughness after fabrication (b).

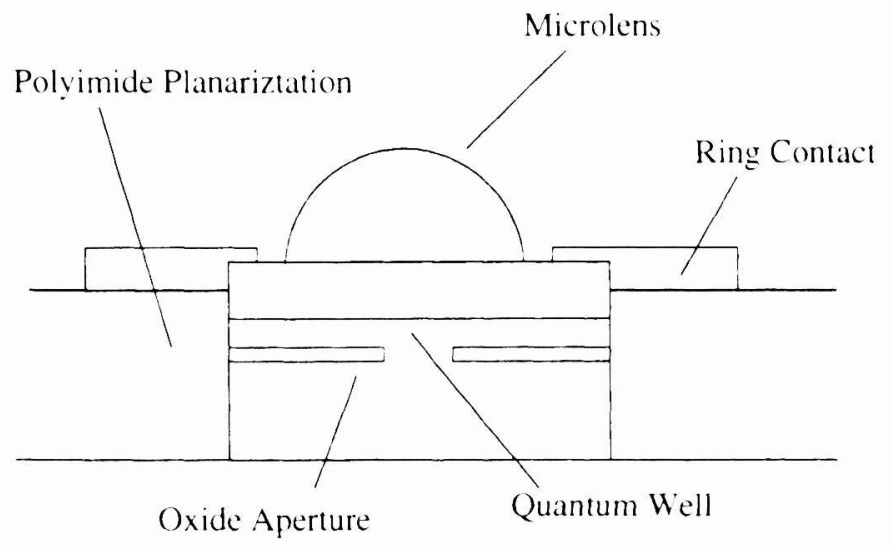

Figure 6. Schematic of an injected self-aligned LED/microlens device 